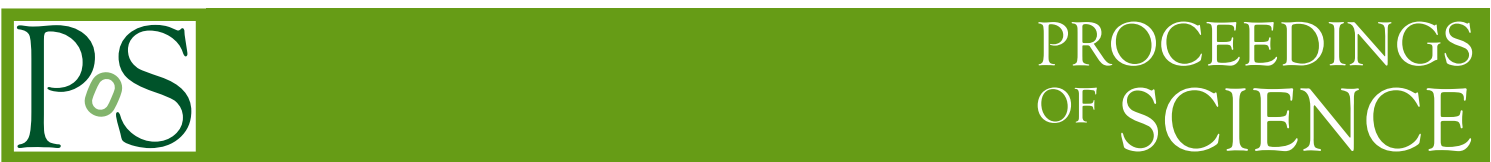

\title{
Hadrons as Holograms
}

\author{
Hilmar Forkel ${ }^{* \dagger}$ \\ Institut für Physik, Humboldt-Universität zu Berlin, D-12489 Berlin, Germany \\ E-mail: forkel@physik.hu-berlin.de
}

\begin{abstract}
We review our recent work on four topics in strong-interaction physics from the perspective of the gauge/gravity correspondence. In particular, we discuss (i) the construction of the "metric soft wall" dual for holographic QCD which reproduces the observed linear square-mass trajectories of radially and orbitally excited (light-quark) hadrons, (ii) an extension of the metric soft wall which encodes diquark correlations holographically and additionally leads to an excellent description of the empirical nucleon excitation spectrum, (iii) an AdS/QCD dual that emerges as a new solution of $5 \mathrm{~d}$ Einstein-dilaton gravity with a specifically derived potential and realizes the area law of the Wilson loop and (approximately) linear meson trajectories dynamically, and finally (iv) the calculation of glueball correlation functions and decay constants in both the hard- and dilaton soft-wall gravity duals, as well as a quantitative analysis of their QCD-relevant physics content.
\end{abstract}

International Workshop on QCD Greens Functions, Confinement and Phenomenology September 7-11, 2009

ECT Trento, Italy

\footnotetext{
${ }^{*}$ Speaker.

${ }^{\dagger}$ Supported by the Deutsche Forschungsgemeinschaft.
} 


\section{Introduction}

The gauge/string correspondence [1] continues to supply new and exciting perspectives for nonperturbative QCD. By holographically relating strongly coupled gauge theories to physically equivalent but weakly coupled string theories, it has provided new analytical tools which promise to eventually describe large- $N_{c}$ QCD in terms of a dual, classical string dynamics [2]. In order to persue the long-term goal of determining this dynamics, current bottom-up approaches, often referred to as "AdS/QCD", construct approximate holographic duals by incrementally encoding known QCD properties (experimental and lattice data, low-energy theorems, the operator product expansion etc.) into $5 \mathrm{~d}$ gravity backgrounds. After implementing the most fundamental features, i.e. conformal symmetry breaking, mass gap, quark confinement etc., one then increasingly incorporates information from the hadron spectrum and from more detailed amplitudes, and sets out to find the gravitational dynamics which generates the obtained background as a solution.

The foundational ingredient of any AdS/QCD dual is the metric of its bulk spacetime. This geometry contains a five-dimensional, non-compact part that takes the generic form of an IRdeformed anti-de Sitter space $\operatorname{AdS}_{5}(R)$ of curvature radius $R$ [3],

$$
d s^{2}=g_{M N}^{\left(\mathrm{AAdS}_{5}\right)} d X^{M} d X^{N}=e^{2 A(z)} \frac{R^{2}}{z^{2}}\left(\eta_{\mu v} d x^{\mu} d x^{v}-d z^{2}\right),
$$

where $\eta_{\mu \nu}$ is the four-dimensional Minkowski metric of the $3+1$ dimensional boundary on which the gauge theory is defined. For an UV-conformal gauge theory like QCD, the metric has to approach $\mathrm{AdS}_{5}$ near the boundary. Since $A \neq 0$ breaks conformal invariance explicitly, this amounts to requiring that $A(z) \rightarrow 0$ as $z \rightarrow 0$. A minimal way of implementing the most crucial IR effects, in particular conformal symmetry breaking and linear quark confinement, is to impose a boundary condition on the string modes at the IR brane of the "hard-wall" metric [3]

$$
e^{2 A_{\mathrm{hw}}(z)}=\theta\left(z_{m}-z\right), \quad z_{m}=\Lambda_{\mathrm{QCD}}^{-1}
$$

where $z_{m}$ acts as an infrared cutoff on the fifth dimension and generates both the mass gap and discrete hadron spectra. The rather drastic hard-wall approximation underlied all of the first bottom-up

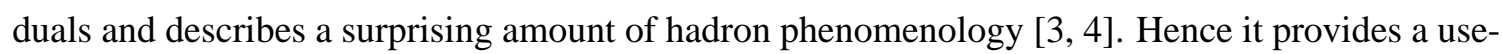
ful benchmark for the development of improved holographic duals. The main limitations of the current generation of AdS/QCD gravity duals are discussed e.g. in Refs. [5], 6].

\section{The "metric soft-wall" dual and linear baryon trajectories}

One of the most prominent and pervasive patterns in the known hadron spectrum consists of linear Regge-type trajectories

$$
M^{2}=M_{0}^{2}+W(N+L)
$$

with approximately universal slopes $W \sim 1.1 \mathrm{GeV}^{2}$ (for the light-quark mesons and baryons) [7] on which the square masses $M^{2}$ of excited states organize themselves in Chew-Frautschi plots, i.e. as a function of both angular momentum $L$ (or alternatively total spin $J$ ) and radial excitation level $N$. The QCD-based understanding of these trajectories and their relation to linear quark confinement remains one of the pre-eminent challenges of strong-interaction physics. 
A serious limitation of holographic duals based on the hard wall (1.2) is that they predict quadratic instead of linear square-mass trajectories as a function of $J, L$ and $N$ (in the gravity approximation) [ []. The first proposal for correcting this shortcoming, the "dilaton soft wall" dual [8], generates linear Regge trajectories $m_{N, J}^{2} \sim N+J$ only in the meson but not in the baryon sector. Baryon trajectories are similarly pronounced in the experimental data [7], however, which led us to construct the "metric soft wall" [9], the so far only AdS/QCD dual which predicts linear trajectories in the baryon sector as well. It further shows that (and partially explains why) universal-slope trajectories (2.1) can be encoded solely into IR deformations $A(z)$.

The metric soft-wall dual is constructed with the help of those string mode fluctuations in the general geometry (1.1) which are dual to the hadronic states under consideration. Casting the wave equations for the "radial" components of the (normalizable) dual bulk modes into the form of Sturm-Liouville eigenvalue problems, one has

$$
\left[-\partial_{z}^{2}+V_{\mathrm{M}}(z)\right] \varphi_{\mathrm{M}}(z)=M_{\mathrm{M}}^{2} \varphi_{\mathrm{M}}(z)
$$

for the modes dual to spin- $0(\mathrm{M}=\mathrm{S})$ and spin-1 $(\mathrm{M}=\mathrm{V})$ mesons as well as

$$
\left[-\partial_{z}^{2}+V_{\mathrm{B}, \pm}(z)\right] \psi_{ \pm}(z)=M_{\mathrm{B}}^{2} \psi_{ \pm}(z)
$$

from the iterated equation for the chirally decomposed Dirac field

$$
\Psi(x, z)=\int \frac{d^{4} k}{(2 \pi)^{4}} e^{-i k x}\left[\psi_{+}^{(k)}(z) P_{+}+\psi_{-}^{(k)}(z) P_{-}\right] \hat{\Psi}^{(4)}(k)
$$

$\left(P_{ \pm} \equiv\left(1 \pm \gamma^{5}\right) / 2\right)$ dual to spin-1/2 baryons (where $\hat{\Psi}^{(4)}$ solves the $4 \mathrm{~d}$ boundary Dirac equation) and similarly for $3 / 2$ baryons $\left[\rrbracket\right.$. The eigenvalues $M_{\mathrm{M}, \mathrm{B}}^{2}$ generate the mass spectra of the gauge theory, and the potentials are

$$
\begin{aligned}
& V_{\mathrm{S}}(z)=\frac{3}{2}\left[A^{\prime \prime}+\frac{3}{2} A^{\prime 2}-3 \frac{A^{\prime}}{z}+\frac{5}{2} \frac{1}{z^{2}}\right]+m_{5, S}^{2} R^{2} \frac{e^{2 A}}{z^{2}} \\
& V_{\mathrm{V}}(z)=\frac{3}{2}\left[-A^{\prime \prime}+\frac{3}{2} A^{\prime 2}-3 \frac{A^{\prime}}{z}+\frac{1}{2} \frac{1}{z^{2}}\right]+m_{5, V}^{2} R^{2} \frac{e^{2 A}}{z^{2}}
\end{aligned}
$$

as well as

$$
V_{\mathrm{B}, \pm}(z)=m_{5, \mathrm{~B}} R \frac{e^{A}}{z}\left[ \pm\left(A^{\prime}-\frac{1}{z}\right)+m_{5, \mathrm{~B}} R \frac{e^{A}}{z}\right] .
$$

The AdS/CFT boundary condition for the bulk modes at small $z$, which relates them to the twist dimensions $\bar{\tau}_{\mathrm{M}}=L+2, \bar{\tau}_{B}=L+3$ of the dual hadron interpolating operators, is then imposed by adjusting the bulk mode masses as [9]

$$
\begin{aligned}
m_{5, \mathrm{~S}}^{2} R^{2} & =\bar{\tau}_{\mathrm{M}}\left(\bar{\tau}_{\mathrm{M}}-4\right)=L^{2}-4, \\
m_{5, \mathrm{~V}}^{2} R^{2} & =\bar{\tau}_{\mathrm{M}}\left(\bar{\tau}_{\mathrm{M}}-4\right)+3=L^{2}-1, \\
m_{5, \mathrm{~B}} R & =\bar{\tau}_{\mathrm{B}}-2=L+1 .
\end{aligned}
$$

The lightest string modes are associated with the leading twist operators, and therefore with the valence quark content of the low-spin (i.e. spin $0,1 / 2,1$, and 3/2) hadron states [4, 10]. The duals 
of their orbital excitations (which have no counterparts in the supergravity spectra) are identified with fluctuations about the AdS background [ [ , 10]. (This identification is incomplete, however, as long as quark flavor is not explicitly accounted for.)

In order to search for IR deformations $A(z)$ which generate the linear trajectorial (LT) structure (2.1), we first determine the required potentials $V_{\mathrm{M}, \mathrm{B}}^{(\mathrm{LT})}$. They should be rising quadratically with $z$ for $z \rightarrow \infty$ to yield an equidistant spectrum for the higher-lying excitations. The more challenging question is how to obtain a universal slope $W$ in both meson and baryon channels. It turns out that this can be achieved by replacing $\bar{\tau}_{i} \rightarrow \bar{\tau}_{i}+\lambda^{2} z^{2}$ in the pure $\mathrm{AdS}_{5}$ potentials (i.e. Eqs. (2.5) - (2.7) with $A \equiv 0$ ) [9], leading to

$$
V_{\mathrm{M}}^{(\mathrm{LT})}(z)=\left[\left(\lambda^{2} z^{2}+L\right)^{2}-\frac{1}{4}\right] \frac{1}{z^{2}}
$$

(which holds for both spin 0 and 1) and

$$
V_{\mathrm{B}, \pm}^{(\mathrm{LT})}(z)=\left\{(L+1)(L+1 \mp 1)+[2(L+1) \pm 1] \lambda^{2} z^{2}+\lambda^{4} z^{4}\right\} \frac{1}{z^{2}} .
$$

The normalizable solutions of the corresponding eigenvalue problems (2.2) and (2.3) can be found analytically [9]. The eigenvalues

$$
M_{\mathrm{M}}^{2}=4 \lambda^{2}\left(N+L+\frac{1}{2}\right), \quad M_{\mathrm{B}}^{2}=4 \lambda^{2}\left(N+L+\frac{3}{2}\right)
$$

indeed generate the observed trajectories (2.1) with universal slope $W=4 \lambda^{2}$ and a mass gap of order $\sqrt{W}$. They further imply the new relations $M_{\mathrm{M}, 0}^{2}=W / 2, M_{\mathrm{B}, 0}^{2}=3 W / 2$ between the ground state masses and the trajectory slope.

One has now to check whether the potentials (2.11), (2.12) can emerge from stringy fluctuations in a bulk gravity background (1.1). We do this by construction, i.e. by equating the general- $A$ potentials (2.5) - (2.7) to their heuristic counterparts (2.11), 2.12), and by then searching for solutions of the resulting differential equations for $A(z)$ subject to the physical boundary conditions. $A$ priori the existence of such a bulk geometry is far from guaranteed since the potentials 2.11, (2.12) may not result from a boundary gauge theory. This is reflected in the fact that the nonlinear, inhomogeneous differential equations for $A$ may not have physically acceptable solutions.

As we have shown in Ref. [9], however, physically sensible IR deformations $A(z)$ indeed emerge as unique solutions. In the baryon sector, the solution $A_{\mathrm{B}}(z)$ subject to the conformal boundary condition $A_{\mathrm{B}}(0)=0$ can be found analytically (for both chiralities),

$$
A_{\mathrm{B}}(z)=\ln \left(1+\frac{\lambda^{2} z^{2}}{m_{5, \mathrm{~B}} R}\right)=\ln \left(1+\frac{\lambda^{2} z^{2}}{L+1}\right) .
$$

The analogous solutions for $A_{\mathrm{S}}$ in the spin- 0 meson and $A_{\mathrm{V}}$ in the vector meson channel, which were numerically obtained and discussed in Ref. [9], are plotted for $L=0, \ldots, 3$ in Fig. 11. The small- $z$ behavior of these solutions hints at the formation of a two-dimensional condensate and indicates its relevance for linear confinement. The $L$ dependence of the resulting $A(z)$ may be interpreted as describing $L$ dependent stringy quantum fluctuations about the AdS background which deform its metric in an $L$ dependent fashion [9]. The nature of the singularities in several mesonic IR deformations, their relation to the RG flow of the associated QCD interpolators, and possible relations to the color-dielectric QCD vacuum structure are also discussed in Ref. [9]. 

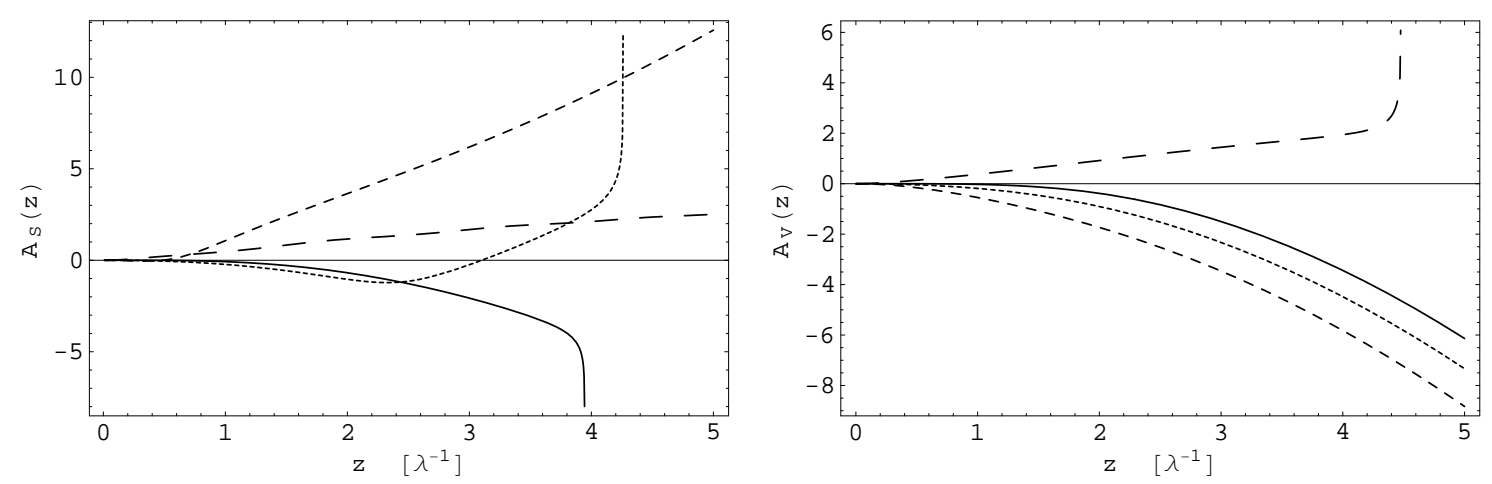

Figure 1: Typical solutions $A_{S}(z)$ (left panel) and $A_{V}(z)$ (right panel) for $L=0$ (full line), $L=1$ (dotted line), $L=2$ (short-dashed) and $L=3$ (long-dashed). The dual eigenmodes have significant support only for $z<\sqrt{2} \lambda^{-1}$.
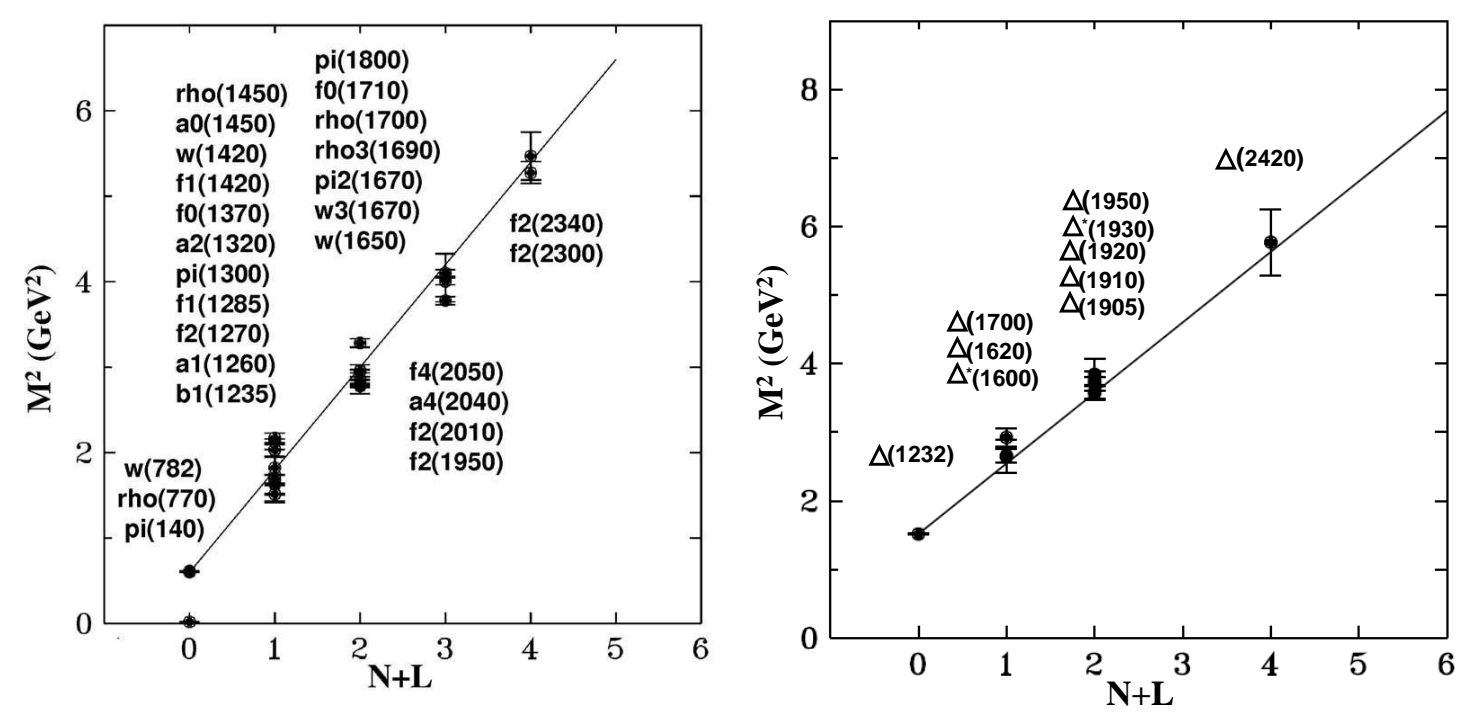

Figure 2: Left panel: Experimental meson mass spectrum from Ref. [11] and the predicted trajectory for $W=2 M_{\rho}^{2} \simeq 1.21 \mathrm{GeV}^{2}$; Right panel: same for the Delta isobar mass spectrum with $S=3 / 2$ (in the ${ }^{4} 8$ representation of $\mathrm{SU}(4))$ and with $W=2 M_{\Delta}^{2} / 3 \simeq 1.01 \mathrm{GeV}^{2}$.

The resulting, overall description of the excited hadron spectra [12] is surprisingly accurate [9]. Using the experimental rho meson mass $M_{\rho}=0.76 \mathrm{GeV}$ [11] to set the deformation scale $\lambda$, the resulting slope $W=1.21 \mathrm{GeV}^{2}$ and spectrum reproduce the experimental meson masses (for quark-antiquark states), as shown in the left panel of Fig. 2. (The pion ground state does not fit into the overall pattern, due to the lack of explicit chiral symmetry.) Alternatively, we can use the experimental $M_{\Delta}$ to determine $\lambda=0.50 \mathrm{GeV}$ which differs by less than $10 \%$ from the value in the meson sector and reflects the approximate slope universality (cf. right panel of Fig. 2). The nucleon excitations require a somewhat smaller value $\lambda=0.47 \mathrm{GeV}$ and are less well described by the trajectory (2.13) which overestimates, in particular, the ground-state mass (yielding $M_{\mathrm{N}}=1.16 \mathrm{GeV}$ ). Finally, the estimates $\Lambda_{\mathrm{QCD}} \simeq \sqrt{W / 8} \simeq 0.35 \mathrm{GeV}$ of the QCD scale 
and $\sigma=W /(2 \pi) \simeq 0.88 \mathrm{GeV} / \mathrm{fm}$ for the string tension turn out to be close to the empirical values.

\section{Holographic diquark correlations and the nucleon excitation spectrum}

While the metric-soft-wall prediction (2.13) works remarkably well in the $\Delta$ sector [13] (all observed $\Delta^{*}$ resonance states lie within errors on the predicted trajectory with empirical slope corresponding to $\lambda=0.52 \mathrm{GeV}$ ), the description of the nucleon data is poorer [9]. In the following section we review our recent extension [14] of the metric-soft-wall dual which generates a universal additive correction

$$
\Delta M_{\mathrm{B}, \kappa_{\mathrm{gd}}}^{2}=-2\left(M_{\Delta}^{2}-M_{N}^{2}\right) \kappa_{\mathrm{gd}}
$$

to Eq. (2.13) which solely depends on the resonances' diquark content. The latter enters through the good (i.e. most attractive) diquark fraction $\kappa_{\mathrm{gd}}$ in the space-spin-flavor baryon wavefunction (i.e. $\kappa_{\mathrm{gd}}=0$ for all $\Delta$ and spin-3/2 $N$ resonances, $\kappa_{\mathrm{gd}}=1 / 4$ for the spin-1/2 negative-parity $N$ resonances, and $\kappa_{\mathrm{gd}}=1 / 2$ for nucleons in the ground state). In order to compare Eqs. (2.13), (3.1) to experimental data, one needs to assign intrinsic orbital and spin angular momenta $L$ and $S$ to the observed states. This has been done on the basis of quark model arguments and extensively discussed in Ref. [14]. The correction (3.1) decidedly improves the agreement with all 48 measured nucleon and $\Delta$ masses, beyond any dynamical quark model prediction of the full mass spectrum.

In searching for a transparent origin of the universal mass correction (3.1) in AdS/QCD, one is led to ask how the diquark content of the baryon resonances can enter a holographic description although diquarks and their operators are gauge dependent while only gauge-invariant operators have well-defined dual modes. The answer to this pivotal question lies in the (leading-twist) baryon interpolating fields [15]

$$
\eta_{t}(x)=2\left[\eta_{\mathrm{pd}}(x)+t \eta_{\mathrm{sd}}(x)\right]
$$

of QCD which contain gauge-invariant diquark information through the pseudoscalar diquark operator in $\eta_{\mathrm{pd}}=\varepsilon_{a b c}\left(u_{a}^{T} C d_{b}\right) \gamma^{5} u_{c}$ and the "good" scalar diquark operator in $\eta_{\mathrm{sd}}=\varepsilon_{a b c}\left(u_{a}^{T} C \gamma^{5} d_{b}\right) u_{c}$. (Here we specialize to $N_{c}=3$, as elsewhere in AdS/QCD.) The interpolators (3.2) are expected to have enhanced overlap with nucleon states of equivalent diquark content and are thereby associated with their good-diquark fraction. This manifests itself in $\kappa_{\mathrm{gd}}$ dependent anomalous dimensions $\gamma_{t}\left(\kappa_{\mathrm{gd}}\right)$ of the corresponding interpolators $\eta_{t}\left(\kappa_{\mathrm{gd}}\right)$ which holographically induce mass corrections

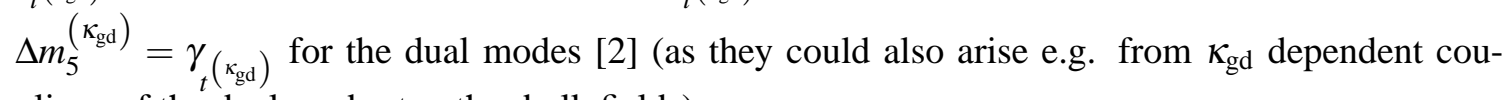
plings of the dual modes to other bulk fields).

In order to include the contributions from the so far neglected anomalous dimensions, we extend the metric soft wall by implementing three bulk spinor fields $\Psi^{\left(\kappa_{\mathrm{gd}}\right)}$ (cf. Eq. (2.4)) dual to the interpolators $\eta_{t}\left(\kappa_{\mathrm{gd}}\right)$ with $\kappa_{\mathrm{gd}}=0,1 / 4$ and $1 / 2$, respectively. These fields are defined as the solutions of the $5 \mathrm{~d}$ Dirac equation with bulk masses

$$
m_{5}^{\left(\kappa_{\mathrm{gd}}\right)}=m_{5}^{(\mathrm{ms})}+\Delta m_{5}^{\left(\kappa_{\mathrm{gd}}\right)}=\frac{L+\Delta m_{5}^{\left(\kappa_{\mathrm{gd}}\right)} R+1}{R}, \quad \kappa_{\mathrm{gd}} \in\{0,1 / 4,1 / 2\}
$$


which ensure that the chirally-odd components $\psi_{-}$satisfy the AdS/CFT boundary conditions. Together with the corresponding IR adjustment

$$
A_{B}(z)=\ln \left(1+\frac{\lambda^{2} z^{2}}{L+\Delta m_{5} R+1}\right)
$$

of the warp factor (2.14), the corrected bulk masses (3.3) were shown in Ref. [14] to generate a universal spectral correction of the form (3.1). In the absence of reliable information on the nonperturbative $\gamma_{t}$ we adjust

$$
\Delta m_{5}^{\left(\kappa_{\mathrm{gd}}\right)}=\frac{\Delta M_{\kappa_{\mathrm{gd}}}^{2}}{4 \lambda^{2} R}
$$

in bottom-up fashion to reproduce the values of (3.1). The eigenvalue spectrum (2.13) then turns into the desired

$$
M_{N, L}^{2}=4 \lambda^{2}\left(N+L+\frac{3}{2}\right)-2\left(M_{\Delta}^{2}-M_{N}^{2}\right) \kappa_{\mathrm{gd}}
$$

(The spectrum (3.6) can also be obtained when the RG flow of the anomalous dimensions, which translates into a $z$ dependent $\Delta m_{5}(z)$, is taken into account [14].) Moreover, the dual modes corresponding to larger $\kappa_{\mathrm{gd}}$ feel the soft wall at smaller $z$ and therefore extend less into the fifth dimension [14]. This reflects the additional attraction in the good-diquark channel and translates into a smaller size of baryons with larger $\kappa_{\mathrm{gd}}$.

\section{Dynamical AdS/QCD}

The AdS/QCD duals discussed above share with most of those so far considered the shortcoming that they are not solutions of a dual gravity. Hence the dynamics which shapes the sought-after QCD dual remains obscure. Some of the present dual candidates, including the dilaton soft-wall of Ref. [8], furthermore fail to exhibit the area-law behavior of the Wilson loop which implies a linearly confining quark-antiquark potential [16]. Others (including the hard wall (1.2)) also confine magnetic charges instead of screening them [17].

In Ref. [16] we have shown how the above limitations can be overcome, by deriving a confining AdS/QCD background from five-dimensional Einstein-dilaton gravity

$$
S=\frac{1}{2 \kappa^{2}} \int d^{5} x \sqrt{|g|}\left(-R+\frac{1}{2} g^{M N} \partial_{M} \Phi \partial_{N} \Phi-V(\Phi)\right)
$$

with a metric restricted to the form (1.1) and a still general potential $V$ for the dilaton $\Phi(z)$. More specifically, we search for static solutions of the corresponding field equations for the background fields $A$ and $\Phi$, which we cast into the form

$$
\Phi^{\prime}=\sqrt{3} \sqrt{-A^{\prime \prime}(z)+A^{\prime 2}(z)+\frac{2}{z} A^{\prime}(z)+\frac{2}{z^{2}}}
$$

and

$$
V(\Phi(z))=-\frac{3 e^{-2 A}}{2 z^{2}}\left[A^{\prime \prime}(z)+3 A^{\prime 2}(z)+\frac{6}{z} A^{\prime}(z)+\frac{2}{z^{2}}\right] .
$$


Our strategy is to construct solutions for the dilaton field $\Phi$ and potential $V(\Phi)$ after prescribing an area-law generating IR deformation $A$. More specifically, we adopt

$$
A(z)=-\frac{1+\sqrt{3}}{2 S+\sqrt{3}-1} \frac{\left(z \Lambda_{\mathrm{QCD}}\right)^{2}}{1+e^{\left(1-z \Lambda_{\mathrm{QCD}}\right)}}
$$

which generates a discrete spectrum with a mass gap and the area law while keeping the fifth dimension non-compact to allow for linear Regge trajectories. Eq. (4.4) remains close to $\mathrm{AdS}_{5}$ in the UV but deforms rather rapidly for $z \gtrsim \Lambda_{\mathrm{QCD}}^{-1}$ to approach the confining large- $z$ asymptotics $A(z) \rightarrow z^{2}$. (The spin dependent factor is required by universality. For a physical interpretation see Ref. [9].) We then find the corresponding dilaton field and potential numerically such that their combination solves the above Einstein-dilaton equations.

The ansatz (4.4) is furthermore designed to generate (approximately) linear Regge trajectories in the highly excited meson spectrum. This spectrum is derived in the tensor gauge-field framework of Ref. [8] which leads to a spin-dependent string-mode potential

$$
\mathscr{V}_{S}(z)=\frac{B^{\prime 2}(z)}{4}-\frac{B^{\prime \prime}(z)}{2}
$$

with $B=-(2 S-1)(\ln z+A)+\Phi$. Important qualitative aspects of the meson spectrum can be understood by studying the UV (i.e. $z \rightarrow 0)$ and IR $(z \rightarrow \infty)$ limits of the mode potential [16]. In Fig. (3) the resulting spectrum is compared to experimental data and hard- and dilaton-soft-wall predictions. A satisfactory description of the meson spectrum with nearly linear trajectories of universal slope is indeed achieved without tuning adjustable parameters, as testified by the rather accurate parametrization

$$
m_{n, S}^{2} \simeq \frac{1}{10}(11 n+9 S+2), \quad(n \geq 1)
$$

(in units of $\mathrm{GeV}$, for $\Lambda_{\mathrm{QCD}}=0.3 \mathrm{GeV}$ ) which makes the approximate slope universality explicit.
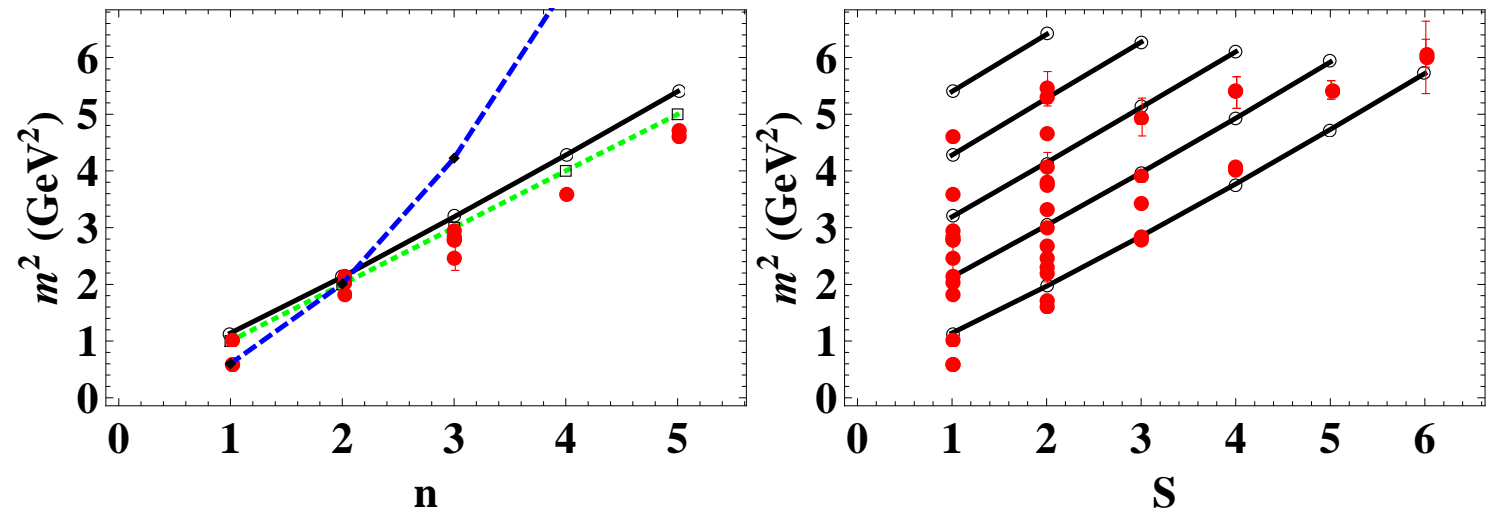

Figure 3: (a) Radial excitations of the rho meson in the hard-wall (dashed line), dilaton-soft-wall [8] (dotted line) and our dynamical soft-wall (solid line, for $\Lambda_{\mathrm{QCD}}=0.3 \mathrm{GeV}$ ) backgrounds. (Note that $n=1$ refers to the nodeless radial ground state.) (b) Square mass predictions of spin excitations vs. the PDG values [11].

Asymptotic freedom and the perturbative corrections to it could additionally be implemented into Eq. (4.4) for small $z \ll \Lambda_{\mathrm{QCD}}^{-1}$, according to the perturbative QCD $\beta$ function. This generates a leading correction $A_{\text {pert }}(z)=(2 \ln z)^{-1}$ which naturally coexists with confinement at large $z$. 


\section{Holographic glueball correlators}

In order to make progress with the construction of improved AdS/QCD duals, one eventually has to analyze more complex and detailed amplitudes. A natural choice are correlation functions of hadronic interpolators which are both directly accessible from the AdS/CFT dictionary and in several cases well-studied in QCD. With this motivation in mind, we have recently derived and analyzed the predicitions of two popular AdS/QCD duals, the hard-wall (1.2) and dilaton soft-wall [8] backgrounds, for the $0^{++}$glueball correlation function and decay constants [6]. Since this work was reviewed in Ref. [18], we will restrict ourselves here to a brief summary. (For related work in the dilaton soft-wall at finite temperature see Ref. [19].)

Both holographic duals turn out to complement each other in their representation of specific nonperturbative glueball physics (at momenta larger than the QCD scale): the soft-wall correlator

$$
\begin{aligned}
\hat{\Pi}^{(\mathrm{sw})}\left(Q^{2}\right)= & -\frac{2 R^{3}}{\kappa^{2}} \lambda^{4}\left[1+\frac{Q^{2}}{4 \lambda^{2}}\left(1+\frac{Q^{2}}{4 \lambda^{2}}\right) \psi\left(\frac{Q^{2}}{4 \lambda^{2}}\right)\right] \\
& \stackrel{Q^{2} \gg \lambda^{2}}{\longrightarrow}-\frac{2}{\pi^{2}} Q^{4}\left[\ln \frac{Q^{2}}{\mu^{2}}+\frac{4 \lambda^{2}}{Q^{2}} \ln \frac{Q^{2}}{\mu^{2}}+\frac{2^{2} 5}{3} \frac{\lambda^{4}}{Q^{4}}-\frac{2^{4}}{3} \frac{\lambda^{6}}{Q^{6}}+\frac{2^{5}}{15} \frac{\lambda^{8}}{Q^{8}}+\ldots\right]
\end{aligned}
$$

(where $\psi(z)=\Gamma^{\prime}(z) / \Gamma(z), \lambda$ is the dilaton mass scale and $R^{3} / \kappa^{2}=2\left(N_{c}^{2}-1\right) / \pi^{2}$ ) contains all known types of QCD power corrections, generated both by vacuum condensates and by a hypothetical UV gluon mass suggested to encode the short-distance behavior of the static quark-antiquark potential [20], while sizeable exponential corrections as induced by small-scale QCD instantons [21] are reproduced in the hard-wall correlator

$$
\begin{aligned}
\hat{\Pi}^{(\mathrm{hw})}\left(Q^{2}\right)= & \frac{R^{3}}{8 \kappa^{2}} Q^{4}\left[2 \frac{K_{1}\left(Q z_{m}\right)}{I_{1}\left(Q z_{m}\right)}-\ln \frac{Q^{2}}{\mu^{2}}\right] \\
& \stackrel{Q^{2} \gg z_{m}^{-2}}{\longrightarrow}-\frac{2}{\pi^{2}} Q^{4} \ln \frac{Q^{2}}{\mu^{2}}+\frac{4}{\pi}\left[1+\frac{3}{4} \frac{1}{Q z_{m}}+O\left(\frac{1}{\left(Q z_{m}\right)^{2}}\right)\right] Q^{4} e^{-2 Q z_{m}}
\end{aligned}
$$

(where the IR brane is located at $z_{m}$ ). This complementarity generalizes to other hadron channels, allows to relate holographic predictions to specific aspects of the gauge dynamics and suggests to combine the underlying brane- and dilaton-induced IR physics into improved QCD duals.

While the various contributions to the holographic estimates (5.1) and (5.2) have the expected order of magnitude, the signs of the two leading power corrections in Eq. (5.1) are opposite to QCD predictions and violate the factorization approximation to the four-gluon condensate. We have argued that this provides specific evidence for the short-distance physics in the Wilson coefficients to be inadequately reproduced by the strongly-coupled UV dynamics of the gravity duals (beyond the leading conformal logarithm) [6]. (This problem cannot be mended by admixing the UV-subleading solution to the bulk-to-boundary propagator [22] without loosing consistency and predictive power [18].) It remains to be seen whether $\alpha^{\prime}$ corrections, in particular the resummed, local ones which are suggested to reproduce the RG flow of the gauge coupling [17], can generate improved holographic predictions for the power corrections.

Since the QCD Wilson coefficients of the $0^{++}$glueball correlator receive unusually small perturbative and enhanced instanton contributions, the hard-wall correlator may yield the better overall AdS/QCD description. Our holographic estimates of the glueball decay constants, which 
are important for experimental glueball searches, provide further evidence for this expectation. The large hard-wall prediction $f_{S}^{(\mathrm{hw})} \simeq 0.8-0.9 \mathrm{GeV}$ for the ground-state decay constant, in particular, reflects the strong instanton-induced short-distance attraction in the scalar QCD glueball correlator, implies an exceptionally small $0^{++}$glueball size and is indeed close to IOPE sum-rule [21] and lattice [23] results. The absence of instanton contributions in the soft wall with its confinementinduced linear meson trajectories, on the other hand, may suggest that instantons are not directly involved in flux-tube formation.

\section{Summary and conclusions}

We have reviewed our recent work on four topics in holographic QCD. To begin with, we have sketched the construction of the "metric soft wall" dual and discussed its capacity to reproduce the empirical combination of radially and orbitally excited hadron mass spectra into linear trajectories of approximately universal slope. The resulting bulk background is solely based on IR deformations of the AdS metric, encodes dual signatures of linear quark confinement and contains only one adjustable parameter related to the string tension. It so far remains the only AdS/QCD dual which is able to reproduce linear trajectories also in the baryon sector. The predicted spectra, as well as new relations between the $\rho$ and $\Delta$ ground state masses and the slopes of their respective trajectories, are in good overall agreement with experimental data.

The metric-soft-wall predictions for the nucleon and its excitations turn out to be significantly less accurate than those in the meson and $\Delta$ sectors, however. This led us to extend this dual by holographically encoding the diquark content of the light baryon states. The latter is specified by the good-diquark fraction of the corresponding baryon interpolators whose anomalous dimensions are then translated by the AdS/CFT dictionary into dual string-mode mass corrections. After implementing the diquark correlations, the improved metric soft wall reproduces the masses of all 48 observed nucleon and $\Delta$ resonances with far better accuracy than e.g. quark models based on substantially larger parameter sets. The behavior of the corresponding bulk modes further reveals that the sizes of the light-quark baryons decrease when their good-diquark content increases.

Another focus of our work was the search for higher-dimensional gravitational dynamics which are capable of generating approximate holographic QCD backgrounds. In particular, we have derived a new solution of the five-dimensional Einstein-dilaton equations with a specific dilaton potential which generates a confining area law for the Wilson loop and can implement the perturbative running of the gauge coupling. It further encodes linear square-mass trajectories for both radial and spin excitations in the meson sector and reproduces the approximately universal slope of the observed trajectories. The result is a satisfactory, fully dynamical description of the light-flavored natural-parity meson spectrum without adjustable parameters beyond the QCD scale.

In order to study the holographic dynamics in more detail, we have further derived and analyzed the $0^{++}$glueball correlation function and its spectral density in the hard-wall and dilaton soft-wall gravity duals. The resulting expressions were confronted with QCD information from the lattice, the instanton-improved operator product expansion (OPE), low-energy theorems etc. This analysis revealed, in particular, that the soft-wall correlator contains all known types of QCD power corrections (including those generated by an effective UV gluon mass) while the hard-wall correlator exhibits in a complementary fashion large exponential corrections as induced by small-scale 
instantons. The results further show that the comparison of holographic predictions with QCD results at the correlator level provides valuable diagnostic insights into the limitations of the underlying duals and leads to useful suggestions for their improvement. The holographic estimates of OPE Wilson coefficients, in particular, were shown to yield detailed and quantitative information on the extent to which the underlying short-distance physics is contaminated by the strongly-coupled UV regime of bottom-up duals. We have further derived predictions for the glueball decay constants which contain crucial size information and are of direct importance for experimental glueball searches. Remarkably, the strong instanton-induced attraction in the $0^{++}$glueball channel is captured by the hard-wall dual, and its prediction $f_{S}^{(\mathrm{hw})} \simeq 0.8-0.9 \mathrm{GeV}$ for the ground-state decay constant agrees inside errors with instanton-improved sum rule and lattice results.

Several current limitations notwithstanding, we conclude that the amount of QCD dynamics encoded in even the simplest holographic duals is encouraging and indicates that the bottom-up approach may eventually turn into a systematic approximation for QCD.

It is a pleasure to thank Tobias Frederico, Eberhard Klempt, Wayne de Paula and Michael Beyer for their collaboration on different parts of the work reviewed above, and the organizers and participants of QCD-TNT 2009 for a very informative and enjoyable workshop. Financial support from the Fundação de Amparo à Pesquisa do Estado de São Paulo (FAPESP) and the Deutsche Forschungsgemeinschaft (DFG) is also acknowledged.

\section{References}

[1] O. Aharony et al., Large-N field theories, string theory and gravity, Phys. Rep. 323 (2000) 183.

[2] K. Peeters and M. Zamaklar, The string/gauge theory correspondence in QCD, Eur. Phys. J. Special Topics 152 (2007) 113 [arXiv: 0708 . 1502]; S.J. Brodsky and G.F. de Téramond, AdS/CFT and Light-Front QCD, arXiv:0802.0514; J. Erdmenger, N. Evans, I. Kirsch and E. Threlfall, Mesons in gauge/gravity duals - a review, Eur. Phys. J. A 35 (2008) 81 [arXiv: 0711 . 4467]; S.S. Gubser and A. Karch, From gauge-string duality to strong interactions: a Pedestrian's Guide, arXiv:0901.0935.

[3] J. Polchinski and M. J. Strassler, Hard scattering and gauge/string duality, Phys. Rev. Lett. 88 (2002) 031601 [hep-th/0109174].

[4] G. F. de Téramond and S. J. Brodsky, Hadronic spectrum of a holographic dual of QCD, Phys. Rev. Lett. 94 (2005) 0201601; H. Boschi, N. Braga and H. Carrion, Glueball Regge trajectories from gauge/string duality, Eur. Phys. J. C 32 (2004) 529; Phys. Rev. D 73 (2006) 047901; J. Erlich, E. Katz, D. T. Son and M. A. Stephanov, QCD and a holographic model of hadrons, Phys. Rev. Lett. 95(2005) 261602; L. Da Rold and A. Pomarol, Chiral symmetry breaking from five dimensions, Nucl. Phys. B 721 (2005) 79; S. J. Brodsky and G. F. de Téramond, Hadronic spectra and light-front wavefunctions in holographic QCD, Phys. Rev. Lett. 96(2006) 0201601; Phys. Rev. D 77 (2008) 056007.

[5] C. Csaki, M. Reece and J. Terning, The AdS/QCD correspondence: still undelivered, arXiv:0811.3001; E. Kiritsis, Dissecting the string-theory dual of QCD, arXiv:0901.1772.

[6] H. Forkel, Holographic glueball structure, Phys. Rev. D 78 (2008) 025001 [arXiv: 0711.1179 ]; AdS/QCD at the correlator level, PoS (Confinement8) (2008) 184 [arXiv: 0812 . 3881].

[7] A.V. Anisovich, V.V. Anisovich and A.V. Sarantsev, Systematics of $q \bar{q}$ states in the $\left(n, M^{2}\right)$ and $\left(J, M^{2}\right)$ planes, Phys. Rev. D 62 (2000) 051502(R) [hep-ph/ 0003113 ]; E. Klempt, A mass 
formula for baryon resonances, Phys. Rev. C 66 (2002) 058201 [hep-ex/ 0206012 ]; D.V. Bugg, Four sorts of meson, Phys. Rep. 397 (2004) 257 [hep-ex/ 0412045 ].

[8] A. Karch, E. Katz, D.T. Son and M.A. Stephanov, Linear confinement and AdS/QCD, Phys. Rev. D 74 (2006) 015005 [hep-ph/0602229].

[9] H. Forkel, M. Beyer and T. Frederico, Linear square-mass trajectories of radially and orbitally excited hadrons in holographic QCD, JHEP 07 (2007) 077 [arXiv: 0705 . 1857]; Linear meson and baryon trajectories in AdS/QCD, Intl. J. Mod. Phys. E 16 (2007) 2794 [arXiv: 0705.4115$].$

[10] S. J. Brodsky and G. F. de Téramond, Light-front hadron dynamics and AdS/CFT correspondence, Phys. Lett. B 582 (2004) 211 [hep-th/0310227].

[11] C. Amsler et al., Review of particle physics, Phys. Lett. B 667 (2008) 1.

[12] R.L. Jaffe, D. Pirjol and A. Scardicchio, Parity doubling among the baryons, Phys. Rep. 435 (2006) 157 [hep-ph / 0602010 ]; L. Glozman, Restoration of chiral and $U(1)_{A}$ symmetries in excited hadrons, Phys. Rep. 444 (2007) 1 [arXiv:hep-ph/0701081]; P. Bicudo, The large degeneracy of excited hadrons and quark models, arXiv:hep-ph/0703114; S.S. Afonin, Parity doubling in particle physics, arXiv:0704.1639.

[13] E. Klempt, Delta resonances, quark models, chiral symmetry and AdS/QCD, Eur. Phys. J. A 38 (2008) 187 [arXiv:0806.4290].

[14] H. Forkel and E. Klempt, Diquark correlations in baryon spectroscopy and holographic QCD, Phys. Lett. B 679 (2009) 77 [arXiv: 0810 . 2959].

[15] D. Espriu, P. Pascual and R. Tarrach, Baryon masses and chiral symmetry breaking, Nucl. Phys. B 214, 285 (1983).

[16] W. de Paula, T. Frederico, H. Forkel and M. Beyer, Dynamical AdS/QCD with area-law confinement and linear Regge trajectories, Phys. Rev. D 79 (2009) 075019 [arXiv: 0806 . 3830]; Solution of the 5D Einstein equations in a dilaton background model, PoS (LC2008) (2008) 046 [arXiv:0810.2710].

[17] U. Gürsoy and E. Kiritsis, Exploring improved holographic theories for QCD: part I, JHEP 02 (2008) 032 [arXiv: 0707.1324 ]; U. Gürsoy, E. Kiritsis and F. Nitti, Exploring improved holographic theories for QCD: part II, JHEP 02 (2008) 019 [arXiv: 0707 . 1349].

[18] H. Forkel, Glueball correlators as holograms, to be published in the Proceedings of Continuous advances in QCD, Minneapolis (2008), arXiv:0808.0304.

[19] A.S. Miranda, C.A. Ballon Bayona, H. Boschi-Filho and N.R.F. Braga, Black-hole quasinormal modes and scalar glueballs in a finite-temperature AdS/QCD model, arXiv:0909.1790.

[20] K.G. Chetyrkin, S. Narison and V.I. Zakharov, Short-distance tachyonic gluon mass and $1 / Q^{2}$ corrections, Nucl. Phys. B 550 (1999) 353 [hep-ph/9811275].

[21] H. Forkel, Scalar gluonium and instantons, Phys. Rev. D 64 (2001) 034015 [hep-ph / 000500 4]; Direct instantons, topological charge screening and QCD glueball sum rules, Phys. Rev. D 71 (2005) 054008 [hep-ph/0312049]; QCD glueball sum rules and vacuum topology, Proceedings of Continuous advances in QCD, Minneapolis (2006), 383 [hep-ph/ 0608071 ].

[22] P. Colangelo, F. De Fazio, F. Jugeau and S. Nicotri, Investigating AdS/QCD duality through scalar glueball correlators, arXiv:0711.4747.

[23] Y. Chen et al., Glueball spectrum and matrix elements on anisotropic lattices, Phys. Rev. D 73 (2006) 014516 [hep-lat/0510074]. 